\title{
Which Stars Form Black Holes and Neutron Stars?
}

\author{
Michael P. Muno \\ Space Radiation Laboratory, California Institute of Technology, Pasadena, CA 91125
}

\begin{abstract}
I describe the current state of our knowledge of the mapping between the initial masses of stars and the compact objects - particularly neutron stars and black holes - that they produce. Most of that knowledge is theoretical in nature, and relies on uncertain assumptions about mass loss through winds, binary mass transfer, and the amount of mass ejected during a supernovae. Observational constraints on the initial masses of stars that produce neutron stars and black holes is scarce. They fall into three general categories: (1) models of the stars that produced the supernova remnants associated with known compact objects, (2) scenarios through with high mass X-ray binaries were produced, and (3) associations between compact objects and coeval clusters of stars for which the minimum masses of stars that have undergone supernovae are known. I focus on the last category as the most promising in the near term. I describe three highly-magnetized neutron stars that have been associated with progenitors that had initial masses of $>30 \mathrm{M}_{\odot}$, and evaluate the prospects of finding further associations between star clusters and compact objects.
\end{abstract}

Keywords: neutron stars - black holes - supernovae - X-rays: binaries

PACS: $97.60 .-\mathrm{s}$

\section{INTRODUCTION}

In introductory astronomy classes, the fates of stars are generally outlined as follows: stars with initial masses $M_{i}<8 \mathrm{M}_{\odot}$ never burn all their nuclear fuel, and eventually become white dwarfs; stars with $8<M_{i}<25 \mathrm{M}_{\odot}$ produce supernovae that eject most of the mass of the star, and leave behind neutron stars; and stars with $M_{i}>25 \mathrm{M}_{\odot}$ are so massive that during supernovae, much of the mass remains bound to the core, so it falls back and causes them to collapse into black holes. What is not generally appreciated is that this story is based almost entirely on theoretical calculations that contain several uncertain elements; observational data is scarce [30]. Establishing observational constraints on these calculations is important because supernovae are the largest source of mass and energy input into the interstellar medium [43]. Therefore, knowing whether a star will form a neutron star or a black hole affects our understanding of how rapidly galaxies can assemble [57], how the abundances of metals in galaxies increase with time [64], and the amount of warm matter ejected into the intergalactic medium [11].

There are several reasons that predicting whether a massive star will produce a neutron star or a black hole is difficult. First, massive stars lose a significant amount of mass through stellar winds as they evolve, but the rates are still uncertain by factors of several $[41,2]$. This problem is most acute for massive stars that become luminous blue variables, because their evolutionary models do not yet include the possibility that they can lose $\sim 10 \mathrm{M}_{\odot}$ of mass in eruptions like that seen from eta Carina in the late 19th century [60]. Second, a large, but poorly-determined, fraction of massive stars are in O 2007 American Institute of Physics 978-0-7354-0434-2/07/\$23.00 
binaries. Mass transfer between the binary stars can occur as they evolve, which would in turn change the mass of each star when it undergoes a supernova [67]. Third, the exact mechanism that turns a collapsing stellar core into a supernova is still uncertain. In particular, it is unknown to what degree rapid rotation and strong magnetic fields might be increase the amount of mass lost during the supernova explosion $[63,1]$, and consequently the mass left behind in a compact remnant.

One way in which the complexity of this problem is manifest from an observational standpoint is in the zoology of neutron stars [56]. It was once assumed that all neutron stars were born as rapidly-rotating radio pulsars, which are powered by particles that are accelerated in their $B \sim 10^{12} \mathrm{G}$ fields as they spin down. However, it now appears the neutron stars are born with a variety of field strengths and rotation rates. First, a class of isolated X-ray pulsars, consisting of soft gamma repeaters and anomalous X-ray pulsars, appear to be magnetars that are powered by the decay of $>10^{14} \mathrm{G}$ fields [70]. Second, it has been suggested that the planets around the isolated millisecond pulsar PSR $1257+12$ could only have survived if that neutron star was formed in something like its present state, rapdily rotating but with with a weak magnetic field $\left(B \sim 10^{9} \mathrm{G}\right)$ [50]. Finally, several manifestations of neutron stars have not been definitively associated with the above categories, including the central compact objects in supernova remnants [7, 58, 35], the "Magnificent Seven" isolated cooling neutron stars [66, 29], and the rotating radio transients [47]. Understanding how this diversity arises should provide insight into how stellar mass, rotation, and magnetic fields affect the mass and energy ejected during a supernovae.

\section{OBSERVATIONAL CONSTRAINTS}

In principle, there are several ways in which one could determine the initial masses of stars that produced black holes and neutron stars: by modeling the supernova remnants associated with compact objects, by creating scenarios by which high-mass X-ray binaries (HMXBs) formed, and by identifying compact objects in coeval star clusters. Each of these has their own strengths and complications.

In principle, if one has identified a supernova associated with a known compact object, it should be possible to infer the mass of the star that exploded from the masses and abundances of metals in the remnant. The Crab nebula is best modeled as descending from an $8-10 \mathrm{M}_{\odot}$ progenitor, $[54]^{1}$ so it is not surprising that it contains a neutron star in the form of a radio pulsar. Likewise, the abundances in the remnant G292.0+1.8 generally matched those produced by a $25 \mathrm{M}_{\odot}$ progenitor in some models $[33,61]$. This is at the upper end of the boundary at which a star is often considered likely to produce a neutron star, and indeed a radio pulsar is associated with the remnant $[5,34]$. However, Cas $\mathrm{A}$ has been variously modeled as originating from a $20-25 \mathrm{M}_{\odot}$ single progenitor [42], or from a 15-25 $\mathrm{M}_{\odot}$ star that was in a binary [71]. The models are complex and do not yet match all of the available data on supernova remnants. Nonetheless, this technique is promising, because 15 of the 45 supernovae within $5 \mathrm{kpc}$ of Earth

\footnotetext{
1 The more massive progenitor suggested by MacAlpine et al. [44] is now disfavored [e.g., 45 ].
} 
are associated with radio pulsars or X-ray emitting central compact objects [37].

The second method is to generate scenarios through which HMXBs could have formed. More massive stars end their lives first, so one would tend to expect that the mass of the mass donor in the binary would represent a lower limit to the mass of the progenitor to the compact object [19]. Cyg X-1 is the prototypical black hole X-ray binary, and provides striking evidence that black holes can indeed form - it contains a $16 \mathrm{M}_{\odot}$ compact object accreting matter from a $33 \mathrm{M}_{\odot} \mathrm{O} 9$ supergiant [28]. Moreover, GX 301-2 is a neutron star X-ray pulsar in a binary with the $50 \mathrm{M}_{\odot}$ B hypergiant Wray 977 [36]. This could suggest that the most massive stars sometimes form neutron stars instead of black holes [19]. However, Wellstein \& Langer [67] have constructed scenarios in which the neutron star GX 301-2 formed from a $25 \mathrm{M}_{\odot}$ star that transferred most of its mass to its companion. Between these results, $4 U$ 1700-37 contains a 50-60 $\mathrm{M}_{\odot} 06.5$ supergiant in a binary with a $2.4 \mathrm{M}_{\odot}$ compact object of undetermined nature [8]. This technique must be pursued further, because although the uncertainties in how mass transfer proceeds are formidable, HMXBs provide the only constraints on the birth masses of compact objects.

The third method is to identify compact objects that are members of coeval populations of stars, for which the maximum initial masses of stars still present is also a lower limit on the masses of stars that have undergone supernovae. Unfortunately, neutron stars and black holes may receive kicks at birth [51, 32], so there is only a short window of time in which one can expect to find a compact object still associated with a star cluster. In rare cases, it may be possible to trace the three-dimensional motions of compact objects back to their birth clusters. This has been attempted for Geminga, for which the proper motion was measured, and the line-of-sight velocity was estimated by modeling the bow shock produced by the pulsar wind nebulae. It was suggested that Geminga formed in the Cas-Tau OB or Ori OB 1a associations, which would imply that its progenitor was $<15 \mathrm{M}_{\odot}$ [55].

Fortunately, in recent years several more straightforward examples have emerged: three magnetars have been found to reside in massive young star clusters $[23,65,52]$. These provide unambiguous lower limits on the masses of progenitors neutron stars, and the results are surprising. I describe these below, focusing on the most clear result of the group, the identification of a slow X-ray pulsar in the Galactic star cluster Westerlund 1.

\section{MAGNETARS IN STAR CLUSTERS}

Among the star clusters associated with magnetars, Westerlund 1 is the most wellstudied (Fig. 1). The cluster contains 24 Wolf-Rayet stars, 4 red supergiant, 4 yellow hypergiants, over $80 \mathrm{OB}$ supergiants, and numerous main-sequence stars as early as $\mathrm{O} 7$ $[68,9]$. The cluster contains $\sim 10^{5} \mathrm{M}_{\odot}$ of stars in a region $6 \mathrm{pc}$ across $(D=5 \mathrm{kpc})$, and is coeval with an age of 4-5 Myr [10]. It is therefore just old enough for $>40 \mathrm{M}_{\odot}$ stars to have undergone supernovae, with a rate of approximately 1 per 10,000 years.

A group of us observed the cluster with Chandra hoping to find black hole HMXBs, but instead we found a $10.6 \mathrm{~s}$ X-ray pulsar, CXOU J164710.2-455216 (Fig. 2) [52, 59]. Although we have not yet measured the spin-down rate for the pulsar $\left(\dot{P}<9 \times 10^{-12}\right.$ $\left.\mathrm{s} \mathrm{s}^{-1}\right)[53,27]$, it exhibits all of the other properties of a magnetar [70]. First, its 




FIGURE 1. I band Image of Westerlund 1 taken with the ESO 2.2m MPG+WFI (copyright ESO). The faintest cluster members in this image are O7 V stars [9].

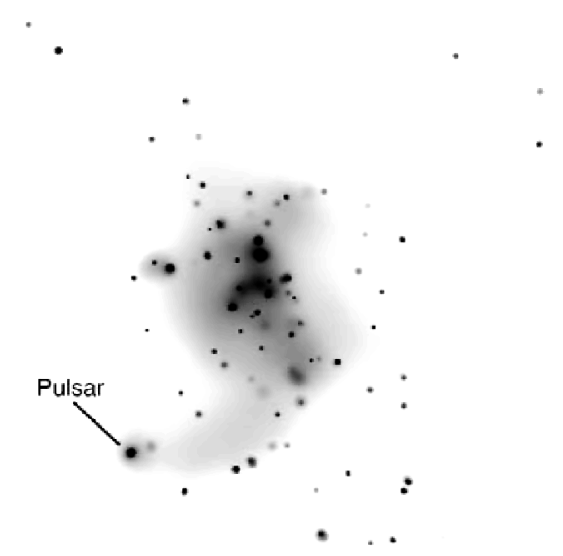

FIGURE 2. Chandra image of Westerlund 1 , with the location of the $10.6 \mathrm{~s}$ X-ray pulsar marked [52].

persistent luminosity is $3 \times 10^{33} \mathrm{erg} \mathrm{s}^{-1}(0.5-8.0 \mathrm{keV})$, which is on the faint end of the range for magnetars. However, the X-ray luminosity is still larger than the upper limit to the spin-down power, so it is not a radio pulsar. Second, the spectrum of CXOU J164710.2-455216 in quiescence can be modeled as a $0.6 \mathrm{keV}$ blackbody originating from $0.1 \%$ of the stellar surface, which is inconsistent with the emission from an ordinary $\left(B \sim 10^{12} \mathrm{G}\right)$ cooling neutron star. Third, there is no infrared counterpart down to $K>18.5$, which precludes its membership in a binary with a companion more massive than a $1 \mathrm{M}_{\odot}$ pre-main-sequence star. Finally, and most conclusively, the Burst Alert Telescope on Swift recently detected a soft gamma-ray burst from CXOU J164710.2-455216 with a fluence of $10^{38} \mathrm{erg}$ and a duration of $20 \mathrm{~ms}$ [39]. This was followed by an increase 


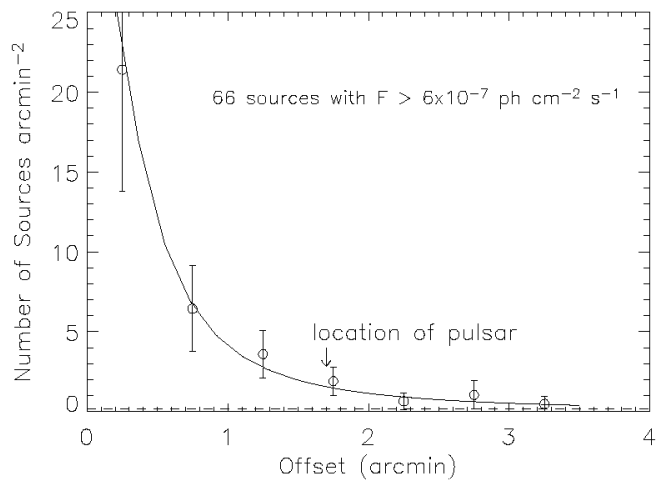

FIGURE 3. The radial distribution of X-ray sources in Westerlund 1 . The dashed line denotes the expected surface density of sources in the Galactic plane [52].

in the persistent luminosity of the pulsar by a factor of 100 , to $1 \times 10^{35} \mathrm{erg} \mathrm{s}^{-1}(0.5-8.0$ $\mathrm{keV})$ [6]. Such an outburst is a hallmark of a magnetar [26, 69].

CXOU J164710.2-455216 is almost certainly member of Westerlund 1. It is located only $1.5^{\prime}$ from the center of the cluster, and based on other observations of the Galactic plane we would expect that $<10 \%$ of X-ray sources at this offset are random interlopers (Fig. 3). Moreover, through another collaboration (A. Nechita, B. Gaensler, et al., in prep.), we have searched 300 archival Chandra and $X M M$ observations of the Galactic plane $\left(|b|<5^{\circ}\right)$ for new X-ray pulsars, and found no new examples with periods between 5-30 s. If we use this to establish an upper limit to the surface density of magnetars toward the Galactic plane, we find that there is a $<0.5 \%$ chance of finding a new example in any given Chandra observation. Therefore, with $99.95 \%$ confidence, CXOU J164710.2-455216 is a member of Westerlund 1, and the progenitor of the pulsar had an initial mass of $>40 \mathrm{M}_{\odot}$.

Similar results have been obtained for three other magnetars. SGR 1806-20 is a member of a star cluster containing at least 4 carbon-type Wolf Rayet stars, 3 OB supergiants, and a luminous blue variable $[23,18,21] .{ }^{2}$ Red supergiants have not yet formed, which suggests that the cluster is 3.0-4.5 Myr old, and that the progenitor to the magnetar had an initial mass of $>50 \mathrm{M}_{\odot}$. Likewise, SGR $1900+14$ is a member of an anonymous star cluster that is probably $<20 \mathrm{Myr}$ old [65], which would place a lower limit of $12 \mathrm{M}_{\odot}$ on the initial mass of its progenitor. However, further studies of both clusters are warranted, because in neither case has the main sequence been identified.

Finally, the AXP 1E 1048.1-5937 lies near the center of a shell of neutral hydrogen emission, which resembles the wind-blown bubbles produced by $30-40 \mathrm{M}_{\odot}$ stars [25].

\footnotetext{
${ }^{2}$ There has been some debate as to the distance to the magnetar, and therefore whether it is a member of the star cluster $[4,48]$. However, such clusters of post-main sequence stars are extremely rare, so it seems unlikely that it is a chance association.
} 
There is debate as to whether the shell and the pulsar are at the same distance [15], but if they are associated, than this AXP would also have had a very massive progenitor.

\section{MASSIVE STARS AND MAGNETARS}

If further evidence accumulates that magnetars appear have $>30 \mathrm{M}_{\odot}$ progenitors, it would have implications for both the Galactic population of these unusual neutron stars, and for the evolution and deaths of massive stars. First, if magnetars only descend from massive progenitors, then they would be expected to be rare compared to radio pulsars [25]. For a standard initial mass function with $N(>M) \propto M^{-1.3}$ [40], only $20 \%$ of stars with $M>8 \mathrm{M}_{\odot}$ have $M>25 \mathrm{M}_{\odot}$. If half of the massive stars produce black holes and half produce magnetars, it would explain why the birth rates of magnetars are only $\sim 10 \%$ of those of radio pulsars $[38,24,25]$.

Second, it implies that some mechanism allows $>30 \mathrm{M}_{\odot}$ stars to lose $>95 \%$ of their mass and leave behind neutron stars. This could occur while the stars evolve, either through powerful stellar winds produced by stars with at least solar metallicity [30], or through mass transfer onto a binary companion [67]. If the mass loss is caused by stellar winds, then one might expect that the majority of $>30 \mathrm{M}_{\odot}$ stars interior to the Solar Circle will form neutron stars instead of black holes (and one might be puzzled by the presence of SGR 0525-66 in the metal-poor LMC). If the binary hypothesis is correct, then the extreme mass loss required to turn a $>30 \mathrm{M}_{\odot}$ star into a neutron star should occur infrequently. Alternatively, some process during the supernova, such as a strong magnetically-driven wind [1], might cause the extreme mass loss. In this case, one must understand how supernovae are triggered before a prediction can be made about the number of magnetars.

Finally, the fact that several magnetars had $>30 \mathrm{M}_{\odot}$ progenitors suggests that there could be a link between massive stars and the presence of strong magnetic fields in their remnants. However, it is not clear when the strong fields of magnetars are generated. One possibility is that they are produced by a dynamo that forms when the core of a rapidly-rotating star collapses [62]. In this case, a link with massive stars could result if they leave cores that rotate more rapidly, possibly because they evolve so rapidly through the red supergiant phase that there is too little time for angular momentum to be lost to the expanding envelope of the star [31]. This scenario would predict that magnetars are produced by the majority of massive stars. Another is that the $>10^{14} \mathrm{G}$ fields are simply those of the progenitor, which were compressed down to the volume of a neutron star during the collapse that ended the star's life [20]. This hypothesis is motivated by the realization that at least three OB stars, $\theta^{1}$ Ori $\mathrm{C}$, HD 191612, and $\tau \mathrm{Sco}$, are highlymagnetized $(\sim 1 \mathrm{kG})[12,13,14]$. In this case, one would expect that the birth rate of magnetars would be proportional to the fraction of $\mathrm{OB}$ stars that are highly-magnetized.

\section{MAKING FURTHER PROGRESS}

Currently, the magnetars identified in star clusters provide the most reliable constraints on the initial masses of stars that produce neutron stars. However, this sample is small, 
largely because the sample of known Galactic star clusters is based on optical observations, and so interstellar extinction makes the sample incomplete beyond $\sim 1 \mathrm{kpc}$ of Earth. Therefore, we (PI: D. Figer) are performing infrared spectroscopy of candidate star clusters taken from the 2MASS [16, 17] and Spitzer/GLIMPSE surveys [49], in order to identify more distant, massive clusters that are likely to harbor compact objects [22]. As the massive clusters are identified, we are searching them in the radio for pulsars, and in X-rays for young, cooling neutron stars and compact objects accreting from the winds of $\mathrm{OB}$ companions. With a larger sample of compact objects in star clusters, we will be able to address better which stars produce black holes and neutron stars, and why.

\section{ACKNOWLEDGMENTS}

I would like to thank J. S. Clark, S. Eikenberry, D. Figer, and B. Gaensler for many discussions, which served as the basis of this review.

\section{REFERENCES}

1. Akiyama, S. \& Wheeler, J. C. 2005, ApJ, 629, 414

2. Bouret, J.-C., Lanz, T., \& Hiller, D. J. 2005, $A \& A, \mathbf{4 3 8}, 301$

3. Burrows, A., Levine, E., Dessart, L., Ott, C. D., \& Murphy, J. 2006, ApJ, 640, 878

4. Cameron, P. B. et al. 2005, Nature, 434, 1112

5. Camilo, F., Manchester, R. N., Gaensler, B. M., Lorimer, D. R., \& Sarkissian, J. 2002, ApJ, 567, L71

6. Campana, S. \& Israel, G. L. 2006, Astronomer's Telegram \#893

7. Chakrabarty, D., Pivovaroff, M. J., Hernquist, L. E., Heyl, J. S., \& Narayan, R. 2001, ApJ, 548, 800

8. Clark, J. S., Goodwin, S. P., Crowther, P. A., Kaper, L., Fairbairn, M., Langer, N. \& Brocksopp, C. 2002, $A \& A, 392,909$

9. Clark, J. S., Negueruela, I., Crowther, P. A., \& Goodwin, S. P. 2005, A\&A, 434, 949

10. Crowther, P. A., Hadfield, L. J., Clark, J. S., Negueruela, I., \& Vacca, W. D. 2006, MNRAS, 372, 1407

11. De Lucia, G., Kauffmann, G., \& White, S. D. M. 2004, MNRAS, 349, 1101

12. Donati, J.-F., Babel, J., Harries, T. J., Howarth, I. D., Petit, P., \& Semel, M. 2002, MNRAS, 333, 55

13. Donati, J.-F., Howarth, I. D., Bouret, J.-C., Petit, P., Catala, C., \& Landstreet, J. 2006a, MNRAS, 365 , L6

14. Donati, J.-F. et al. 2006b, $M N R A S, 370,629$

15. Durant, M. \& van Kerkwijk, M. H. 2006, ApJ, 650, 1070

16. Dutra, C. M., \& Bica, E. 2001, A\&A, 376, 434

17. Dutra, C. M., Bica, E., Soares, J., \& Barbuy, B. 2003, A\&A, 400, 533

18. Eikenberry, S. S. et al. 2004, ApJ, 616, 506

19. Ergma, E. \& van den Heuvel, E. P. J. 1998, $A \& A, 331$, L29

20. Ferrario, L. \& Wickramasinghe, D. T. 2006, MNRAS, 367, 1323

21. Figer, D. F., Najarro, F., Geballe, T. R., Blum, R. D., \& Kudritzki, R. P. 2005, ApJ, 622, L49

22. Figer, D. F., Mackenty, J. W., Robberto, M., Smith, K., Najarro, F., Kudrittzki, R. P., \& Herrero, A. 2006, ApJ, 646, 1452

23. Fuchs, Y., Mirabel, F., Chaty, S., Claret, A., Cesarsky, C. J., \& Cesarsky, D. A. 1999, $A \& A, 350,891$

24. Gaensler, B. M., Gotthelf, E. V., \& Vasisht, G. 1999, ApJ, 526, L37

25. Gaensler, B. M., McClure-Griffiths, N. M., Oey, M. S., Haverkorn, M., Dickey, J. M., \& Green, A. J. 2005, ApJ, 620, L95

26. Gavriil, F. P., Kaspi, V. M., \& Woods, P. M. 2002, Nature, 419, 142

27. Gavriil, F. P., Woods, P. M., \& Kaspi, V. M. 2006, Astronomer's Telegram \#901

28. Gies, D. R. \& Bolton, C. T. 1986, ApJ, 304, 371 
29. Haberl, F. 2006, astro-ph/0609066

30. Heger, A., Fryer, C. L., Woosley, S. E., Langer, N., \& Hartmann, D. H. 2003, ApJ, 591, 288

31. Heger, A., Woosley, S. E. \& Spruit, H. C. $2005, A p J, 626,350$

32. Hobbs, G., Lorimer, D. R., Lyne, A. G., \& Kramer, M. 2005, MNRAS, 360, 974

33. Hughes, J. P. \& Signh, K. P. 1994, ApJ, 422, 126

34. Hughes, J. P., Slane, P. O., Park, S., Roming, P. W. A., \& Burrows, D. N. 2003, ApJ, 591, L139

35. Hui, C.-Y. \& Becker, W. 2006, $A \& A, 454,543$

36. Kaper, L. Lamers, H. J. G. L. M., Ruymaekers, E., van den Heuvel, E. P J., \& Zuidervijk, E. J. 1995, ApJ, 300, 446

37. Kaplan, D. L., Frail, D. A., Gaensler, B. M., Gotthelf, E. V., Kulkarni, S. R., Slane, P. O., \& Nechita, A. 2004, ApJS, 153, 269

38. Kouveliotou, C. et al. 1994, Nature, 368, 125

39. Krimm, H., Barthelmy, S., Campana, S., Cumming, J., Israel, G., Palmer, D., \& Parsons, A. 2006, Astronomer's Telegram \#894.

40. Kroupa, P. 2002, Science, 295,82

41. Lamers, H. J. G. L. M. \& Leitherer, C. 1998, $A p J, \mathbf{4 1 2}, 771$

42. Laming, J. M. \& Hwang, U. 2003, ApJ, 597, 347

43. Leitherer C., Robert, C., \& Drissen, L. 1992, ApJ, 401, 596

44. MacAlpine, G. M., McGaugh, S. S., Mazzarella, J. M., \& Umoto, A. 1989, ApJ, 342, 364

45. MacAlpine, G. M., Lawrence, S. S., Sears, R. L., Sosin, M. S., \& Henry, R. B. C. 1996, ApJ, 463, 650

46. Markova, N., Puls, J., Repolust, T., \& Markov, H. 2004, $A \& A, 413,693$

47. McLaughlin, M. A. et al. 2006, Nature, 439, 817

48. McClure-Griffiths, N. M. \& Gaensler, B. M. 2005, ApJ, 630, L161

49. Mercer, E. P. et al. 2005, ApJ, 635, 560

50. Miller, M. C. \& Hamilton, D. P. $2001, A p J, 550,863$

51. Mirabel, I. F., Mignani, R., Rodrigues, I., Combi, J. A., Rodríguez, L. F., \& Grugliemetti, F. 2002, $A \& A, \mathbf{3 9 5}, 595$

52. Muno, M. P. et al. 2006a, ApJ, 636, L41

53. Muno, M. P., Gaensler, B., Clark, J. S., Portegies Zwart, S., Pooley, D., de Grijs, R., Stevens, I, \& Negueruela, I. 2006b, Astronomer's Telegram, \#902

54. Nomoto, K., Sugimoto, D., Sparks, W. M., Fesen, R. A., Gull, T. R., \& Miyaji, S. 1982, Nature, 299, 803

55. Pellizza, L. J., Mignani, R. P., Greiner, I. A., \& Mirabel, I. F. 2005, $A \& A, \mathbf{4 3 5}, 625$

56. Popov, S. B., Turolla, R., \& Possenti, A. 2006, MNRAS, 369, L23

57. Scannapieco, C., Tissera, P. B., White, S. D. M., \& Springel, V. 2006, MNRAS, 371, 1125

58. Seward, F. D., Slane, P. O., Smith, R. K., \& Sun, M. 2003, ApJ, 584, 414

59. Skinner, S. L., Perna, R., \& Shekov, S. A. 2006, astro-ph/0607528

60. Smith, N. \& Owocki, S. P. 2006, ApJ, 645, L45

61. Thielemann, F.-K., Nomoto, K., \& Hashimoto, M. 1996, 460, 408

62. Thompson, C. \& Duncan, R. C. 1993, ApJ, 408, 194

63. Thomspon, T. A., Chang, P., \& Quataert, E. 2004, ApJ, 611, 380

64. Tsujimoto, T., Nomoto, K., Yoshii, Y., Hashimoto, M., Yanagida, S., \& Thielemann, F.-K. 1995, MNRAS, 277, 945

65. Vrba, F. J., Henden, A. A., Luginbuhi, C. B., Guetter, H. H., Hartmann, D. H., \& Klose, S. 2000, ApJ, $\mathbf{5 3 3}$, L17

66. Walter, F. M., Wolk, S. J., \& Neuhauser, R. 1996, Nature, 379, 233

67. Wellstein, S. \& Langer, N. 1999, $A \& A, 350,148$

68. Westerlund, B. E. $1987, A \& A S, 70,311$

69. Woods, P. M. et al. 2004, ApJ, 605, 378

70. Woods, P. M. \& Thompson, C. 2006, in Compact Stellar X-ray Source, eds. W. Lewin \& M. van der Klis, Cambridge University Press

71. Young, P. A. et al. 2006, ApJ, 640,891 